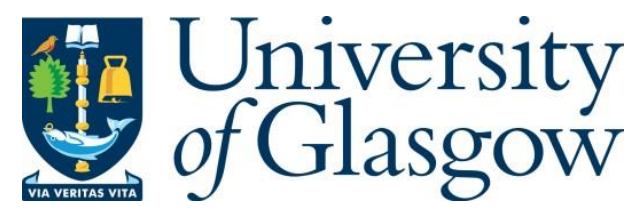

Onireti, O., Héliot, F., and Imran, M. A. (2012) On the Energy Efficiency-Spectral Efficiency Trade-Off of the 2BS-DMIMO System. In: 2012 IEEE Vehicular Technology Conference (VTC Fall), Québec City, Canada, 03-06 Sep 2012, ISBN 9781467318815.

There may be differences between this version and the published version. You are advised to consult the publisher's version if you wish to cite from it.

http://eprints.gla.ac.uk/136915/

Deposited on: 14 February 2017

Enlighten - Research publications by members of the University of Glasgow http://eprints.gla.ac.uk 


\title{
On the Energy Efficiency-Spectral Efficiency Trade-Off of the 2BS-DMIMO System
}

\author{
Oluwakayode Onireti, Fabien Héliot, and Muhammad Ali Imran \\ Centre for Communication Systems Research (CCSR), Faculty of Electronics \& Physical Sciences, \\ University of Surrey, Guildford GU2 7XH, UK. Email: O.Onireti@surrey.ac.uk
}

\begin{abstract}
In this paper, we propose a novel closed-form approximation of the Energy Efficiency vs. Spectral Efficiency (EESE) trade-off for the uplink/downlink of distributed multipleinput multiple-output (DMIMO) system with two cooperating base stations. Our closed-form expression can be utilized for evaluating the idealistic and realistic EE-SE performances of various antenna configurations as well as assessing how DMIMO compares against MIMO system in terms of EE. Results show a tight match between our closed-form approximation and the Monte-Carlo simulation for both idealistic and realistic EESE trade-off. Our results also show that given a target SE requirement, there exists an optimal antenna setting that maximizes the EE. In addition, DMIMO scheme can offer significant improvement in terms of EE over the MIMO scheme.
\end{abstract}

\section{INTRODUCTION}

The traditional approach for designing wireless network focuses on the spectral efficiency (SE) metric for optimizing system performance. The current trend of increasing energy demand and increasing energy related operating cost is currently steering research towards the design of energy efficient networks. However, a conflict of interest does exist between maximizing SE, which is a ratio of the capacity in bits/s to the available spectrum, and maximizing energy efficiency (EE), which is a ratio of the capacity to the total consumed power $P_{T}$ [1]. The SE is the spectrum utilization indicator while the $\mathrm{EE}$ is the energy consumption indicator, hence the relationship between both indicators needs to be carefully studied through their trade-off, i.e. the EE-SE trade-off.

The EE-SE trade-off of the point-to-point additive white Gaussian noise (AWGN) can be easily computed [2]. However, closed-form approximations (CFAs) are required for explicitly expressing the EE-SE trade-off of more complex channel such as point-to-point multiple-input multiple-output (MIMO) Rayleigh fading channel [3], [4] . Furthermore, the CFA of the EE-SE trade-off for the uplink of the symmetric coordinated multi-point (CoMP) system is given in [5]. In this work, we are interested in obtaining a tight CFA for the EESE trade-off of the distributed MIMO system, which is a promising technique for meeting the high data rate requirement of the next generation mobile communication networks. The DMIMO scheme combines both the advantages of point-topoint MIMO and distributed antenna system (DAS), i.e. micro and macro diversity, respectively [6], [7]. In [6], [8]-[10], closed-form expressions of the channel capacity of DMIMO were presented. To the best of our knowledge, the CFA of the EE-SE trade-off of DMIMO is yet to be presented.
In this paper, we present a framework to analyze the EESE trade-off of the DMIMO system with two cooperating base stations (2BS-DMIMO) over the Rayleigh fading channel by following the same approach as in the pioneering works of [3] and [4] on the the EE-SE trade-off CFA for the single-user MIMO scenario. In Section II, we introduce the system model for the 2BS-DMIMO. In Section III, we first derive the CFA of the EE-SE trade-off for the uplink of the 2BS-DMIMO by designing a parametric function and using a heuristic curve fitting method [3], [4], [11]. Then, we derive the CFA of the EE-SE trade-off for the downlink of the 2BS-DMIMO by relying on a similar approach as in the uplink. Numerical results show a tight match of our CFAs with Monte-Carlo simulation for both uplink and downlink scenarios. In Section IV, we utilized our CFAs along with the realistic power models in [12] and [13], to obtain the realistic EE in the downlink of the 2BS-DMIMO and its EE gain against MIMO system over the Rayleigh fading channel. Our results show that there exists an optimal number of BS antennas that maximizes EE and that 2BS-DMIMO can be far more energy efficient than MIMO system.

\section{System MODEL}

We consider a standard DMIMO communication system where two base stations (BSs) equipped with $p$ antennas each cooperate to transmit/receive data to/from a user terminal (UT) equipped with $q$ antennas, as illustrated in Fig. 1. We consider only one active user in the system due to the use of an orthogonal access scheme. We assume as in [6], [9], [14] that all $2 p$ antennas have a separate feeder to the central unit where all signal processing is done. We also assume that $p \geq q$, which is a practical and reasonable assumption [7]. The matrices $\boldsymbol{\Omega}_{i}$ and $\mathbf{H}_{i}$ represent the deterministic distance dependent pathloss/shadowing and the MIMO Rayleigh fading channel, respectively, between the $i^{t h}$ BS and the UT, $i \in\{1,2\}$. The channel model of the DMIMO system which is depicted in Fig. 1 can then be defined as $\tilde{\mathbf{H}}=\mathbf{\Omega} \odot \mathbf{H}$, where $\mathbf{H}=\left[\mathbf{H}_{1}^{\dagger}, \mathbf{H}_{2}^{\dagger}\right]^{\dagger},(.)^{\dagger}$ is the complex conjugate transpose, $\odot$ denotes the Hadamard product, $\tilde{\mathbf{H}} \in \mathbb{C}^{N_{r} \times N_{t}}, \mathbf{H} \in \mathbb{C}^{N_{r} \times N_{t}}$ and $\boldsymbol{\Omega} \in \mathbb{R}_{+}^{N_{r} \times N_{t}}$ with $R_{+}=\{x \in \mathbb{R} \mid x \geq 0\}$. Moreover, $\boldsymbol{\Omega}=\boldsymbol{\Lambda} \triangleq\left[\alpha_{1} \mathbf{J}^{\dagger}, \alpha_{2} \mathbf{J}^{\dagger}\right]^{\dagger}$ and $\boldsymbol{\Omega}=\boldsymbol{\Lambda}^{\dagger}$ in the uplink and downlink cases, respectively, where $\mathbf{J}$ is a $p \times q$ matrix with all elements equal to one and $\alpha_{i}$ represents the average channel gain between the UT and the $i^{\text {th }} \mathrm{BS}$. Furthermore, the total number of transmit and receive antennas of the 2BS-DMIMO 


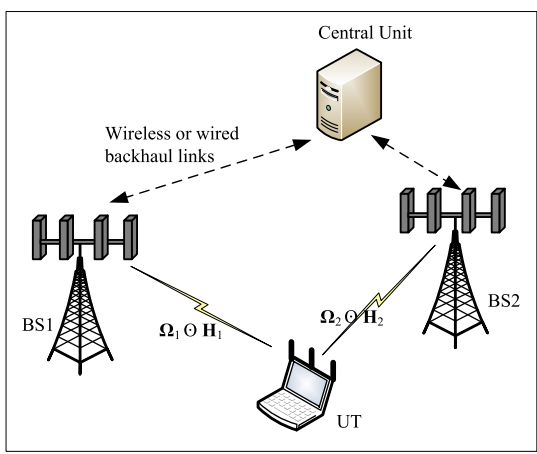

Fig. 1. Distributed MIMO system model (2BS-DMIMO)

system is defined as $N_{t}$ and $N_{r}$, respectively. In the uplink case $N_{t}=n=q$ and $N_{r}=2 p$, whereas in the downlink case $N_{t}=2 p, N_{r}=q$ and $n=p$, where $n$ is the number of transmit antenna per node. The received signal $y \in \mathbb{C}^{N_{r} \times 1}$ can be expressed as

$$
y=\tilde{\mathbf{H}} \mathbf{x}+\mathbf{z}
$$

where $\mathbf{x} \in \mathbb{C}^{N_{t} \times 1}$ is the transmit signal vector with average transmit power $P$ and $\mathbf{z} \in \mathbb{C}^{N_{r} \times 1}$ is the noise vector with average noise power $N$. We assume that $\mathbf{H}$ is a random matrix having independent and identically distributed (i.i.d) complex circular Gaussian entries with zero-mean and unit variance. The maximum achievable SE or ergodic capacity per unit bandwidth of the DMIMO system given in (1) can be expressed from [6] as

$$
C=\mathbb{E}_{\tilde{H}}\left\{\log _{2}\left|\mathbf{I}_{N_{r}}+\frac{\gamma}{n} \tilde{\mathbf{H}} \tilde{\mathbf{H}}^{\dagger}\right|\right\}
$$

where $\mathbf{I}_{N_{r}}$ is a $N_{r} \times N_{r}$ identity matrix, $\mathbb{E}$ is the expectation, $\gamma \triangleq \frac{P}{N_{0} W}$ is the average signal-to-noise ratio (SNR), $W(\mathrm{~Hz})$ is the bandwidth and $N_{0}$ is the noise spectral density.

\section{Closed-Form Approximation of the EE-SE TRADE-OFF}

The capacity per unit bandwidth of the Rayleigh fading DMIMO channel given in (2) is such that

$$
C=f(\gamma) \text {. }
$$

The EE, $C_{J}$ is the bit-per-Joule capacity and is equivalent to $R / P_{T}$, where $R$ is the achievable rate and $P_{T}$ is the total consumed power. Note that when considering the idealistic model, $P_{T}=P$ and $P_{T}=2 P$ in the uplink and downlink cases, respectively. Using the inverse function of $f, f^{-1}$ (i.e. $f_{u}^{-1}$ and $f_{d}^{-1}$ for the uplink and downlink case, respectively), for expressing $\gamma$ as a function of $C$, we obtain that

$$
\begin{aligned}
C_{J_{u}} & =\frac{S}{N_{0} f_{u}^{-1}(C)}, \\
C_{J_{d}} & =\frac{S}{2 N_{0} f_{d}^{-1}(C)},
\end{aligned}
$$

for the uplink and the downlink of the 2BS-DMIMO system, respectively, where $S=R / W$. Equations (4) and (5) indicate that the EE-SE trade-off can be formulated by finding an explicit expression for $f^{-1}(C)$. For example, $f^{-1}(C)$ can easily be obtained for point-to-point AWGN channel as in [2], however, this is not as straightforward for more complex channel scenarios such as DMIMO. Instead, approximating $f^{-1}(C)$ as in [3]-[5] is an effective solution for formulating a closed-form expression of the DMIMO EE-SE trade-off.

\section{A. EE-SE Trade-off CFA for the Uplink of 2BS-DMIMO}

The closed-form expression for the ergodic capacity per unit bandwidth in the uplink of the 2BS-DMIMO system can be expressed from [8] as

$$
\begin{aligned}
C \approx \frac{1}{\ln (2)}[ & q \ln \left(1+\kappa \alpha_{1}^{2} P u_{1}+\kappa \alpha_{2}^{2} P u_{2}\right)+p \ln \left(1+\alpha_{1}^{2} P w\right) \\
& \left.+p \ln \left(1+\alpha_{2}^{2} P w\right)-p\left(\alpha_{1}^{2} P u_{1}+\alpha_{2}^{2} P u_{2}\right) w\right]
\end{aligned}
$$

in bits/s/Hz, where $\kappa=\frac{p}{q} u_{i}, i \in\{1,2\}$ and $w$ are the unique solution to the following equations:

$$
\begin{aligned}
& u_{i}=\left(1+\alpha_{i}^{2} P w\right)^{-1} \quad i=1,2 \\
& w=\left(1+\kappa \alpha_{1}^{2} P u_{1}+\kappa \alpha_{2}^{2} P u_{2}\right)^{-1}
\end{aligned}
$$

Let us define $g=\kappa \alpha_{2}^{2} P u_{2}(\Delta x+1), d_{1}=\Delta \alpha_{2}^{2} P w$ and $d_{2}=\alpha_{2}^{2} P w$, where $\Delta$ is the SNR offset between the two links i.e. $\Delta=\frac{\alpha_{1}^{2}}{\alpha_{2}^{2}}, \alpha_{2}$ is the link with the lowest gain and $x=u_{1} / u_{2}$. In addition, let $\bar{g}=2 g+1, \bar{d}_{1}=2 d_{1}+1$ and $\bar{d}_{2}=2 d_{2}+1$. Then, equation (6) can be re-expressed as

$$
C \approx \tilde{f}_{u}(\gamma)=\frac{1}{\ln (2)}\left(S_{q}+S_{p_{1}}+S_{p_{2}}\right)
$$

where $S_{q}, S_{p_{1}}$ and $S_{p_{2}}$ are given by

$$
\begin{aligned}
S_{q} & =q\left(-\frac{1}{2}-\ln (2)+\frac{1}{1+\bar{g}}+\ln (1+\bar{g})\right) \\
S_{p_{1}} & =p\left(-\frac{1}{2}-\ln (2)+\frac{1}{1+\bar{d}_{1}}+\ln \left(1+\bar{d}_{1}\right)\right) \text { and } \\
S_{p_{2}} & =p\left(-\frac{1}{2}-\ln (2)+\frac{1}{1+\bar{d}_{2}}+\ln \left(1+\bar{d}_{2}\right)\right)
\end{aligned}
$$

respectively. We can re-express the first equation in (9) as

$$
g_{q}\left(S_{q}\right)=-\frac{1}{1+\bar{g}} \exp \left(-\frac{1}{1+\bar{g}}\right)
$$

where $g_{q}\left(S_{q}\right)=-\exp \left(-\left(\frac{S_{q}}{q}+\frac{1}{2}+\ln (2)\right)\right)$. Using the Lambert $W$ function which is the inverse function of $f(w)=w \exp (w)$ and is such that $W(z) e^{W(z)}=z$, where $w, z \in \mathbb{C}$ [15], we can reformulate (10) as

$$
\begin{aligned}
-\frac{1}{1+\bar{g}} & =W_{0}\left(g_{q}\left(S_{q}\right)\right) \\
\bar{g} & =-\left[1+\frac{1}{W_{0}\left(g_{q}\left(S_{q}\right)\right)}\right] .
\end{aligned}
$$

Similarly, $\bar{d}_{1}=-\left[1+\frac{1}{W_{0}\left(g_{p}\left(S_{p_{1}}\right)\right)}\right]$ and $\bar{d}_{2}=-\left[1+\frac{1}{W_{0}\left(g_{p}\left(S_{p_{2}}\right)\right)}\right]$. Moreover, it can be easily demonstrated that

$$
\bar{g}\left[\bar{d}_{2}+\left(\frac{\bar{d}_{1}}{\Delta}-\frac{1}{\Delta}+1\right)\right]=2\left[2 \alpha_{2}^{2} \gamma(\kappa \Delta x+\kappa+1)+1\right],
$$




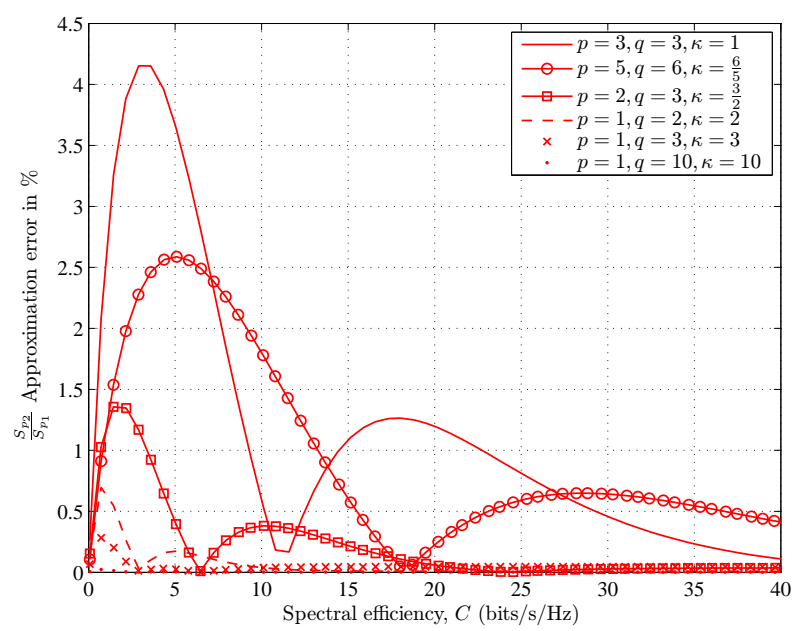

Fig. 2. Accuracy of $\frac{S_{p_{2}}}{S_{p_{1}}}$ in $\%$

which results in the following approximation for $f_{u}^{-1}(C)$ in (4)

$$
\tilde{f}_{u}^{-1}(C)=\frac{-2+\left[1+\frac{1}{W_{0}\left(g_{q}\left(S_{q}\right)\right.}\right]\left[\left(1+\frac{1}{W_{0}\left(g_{p}\left(S_{p_{1}}\right)\right)}\right)+\frac{1}{\Delta}\left(2+\frac{1}{W_{0}\left(g_{p}\left(S_{p_{2}}\right)\right)}\right)-1\right]}{4 \alpha_{2}^{2}(\kappa \Delta x+\kappa+1)}
$$

Note that $u_{1}=2 /\left(1+\bar{d}_{1}\right)$ and $u_{2}=2 /\left(1+\bar{d}_{2}\right)$ are such that

$$
x=\frac{u_{1}}{u_{2}}=\frac{W_{0}\left(g_{p}\left(S_{p_{1}}\right)\right)}{W_{0}\left(g_{p}\left(S_{p_{2}}\right)\right)} .
$$

Thus, obtaining the closed-form expression of the EE-SE trade-off for the uplink of the 2BS-DMIMO system is equivalent to expressing $S_{q}, S_{p_{1}}$ and $S_{p_{2}}$ as a function of $C$ in (13). Moreover, since $\bar{C}=C \ln (2) \approx S_{q}+S_{p_{1}}+S_{p_{2}}$ in (8), we can define parametric functions $\Psi_{p, q}(C) \approx \frac{S_{p_{2}}}{S_{p_{1}}}$ and $\Phi_{p, q}(C) \approx S_{q}-\left(S_{p_{1}}+S_{p_{2}}\right)$, such that we obtain $S_{q}, S_{p_{1}}$ and $S_{p_{2}}$ as a function of $C, p$ and $q$ by solving a set of linear equations. The equation $S_{q}-\left(S_{p_{1}}+S_{p_{2}}\right)$ can be simplified as

$$
\Phi_{p, q}(C) \approx S_{q}-\left(S_{p_{1}}+S_{p_{2}}\right)=\ln \left(\frac{2^{2 p}(1+\bar{g})^{q}}{2^{q}\left(1+\bar{d}_{1}\right)^{p}\left(1+\bar{d}_{2}\right)^{p}}\right),
$$

since it can be proved that $p(-1 / 2+1 /(1+\bar{g}))-q(-1+$ $\left.1 /\left(1+\bar{d}_{1}\right)+1 /\left(1+\bar{d}_{2}\right)\right)=0$ as in the MIMO case [3]. We define $\phi_{p, q}(C)=e^{\frac{\Phi_{p, q}}{q}} \approx e^{\frac{S_{q}-\left(S_{p_{1}}+S_{p_{2}}\right)}{q}}$ and utilize the parametric function derived for $S_{q}-S_{p}$ in the point-to-point MIMO Rayleigh fading channel [3], such that

$$
\begin{aligned}
& \Phi_{p . q}(C)=q \eta_{1} \ln \left(\cosh \left(\bar{C} /\left(q \eta_{1}\right)\right)\right) \text { and } \\
& \Phi_{p, q}(C)=q \ln \left(1+\eta_{0}\left[\cosh \left(\bar{C} /\left(q \eta_{1}\right)\right)^{\eta_{1}}-1\right]\right)
\end{aligned}
$$

tightly approximate $S_{q}-\left(S_{p_{1}}+S_{p_{2}}\right)$ as a function of $C$ when $\kappa \geq 2$ and $1 \leq \kappa<2$, respectively, where $\eta_{0}$ and $\eta_{1}$ are obtained from a numerical search.

In order to obtain $\Psi_{p, q}$, we first numerically evaluate $\frac{S_{p_{2}}}{S_{p_{1}}}$ as a function of $C$ for a particular $\Delta$ and various antenna configurations, we set $\alpha_{1}^{2}=1$ and $\alpha_{2}^{2}=0.1$ i.e., $\Delta=10 \mathrm{~dB}$.

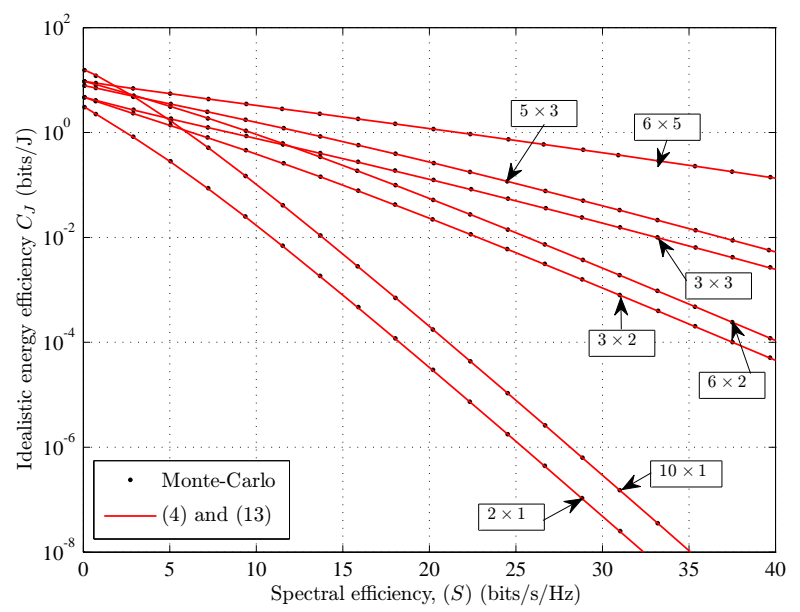

Fig. 3. EE-SE of the Uplink of 2BS-DMIMO

In addition, we obtained that $\lim _{C \rightarrow 0} \frac{S_{p_{2}}}{S_{p_{1}}}=\frac{1}{\Delta}$, such that the parametric function that best fits the curves $\frac{S_{p_{2}}}{S_{p_{1}}}$ as a function of $C$ is

$$
\Psi_{p, q}=\frac{1}{\Delta}+\ln \left(1+\delta_{1} \tanh \left(\bar{C} \delta_{0} / n\right)\right),
$$

where the values of $\delta_{0}$ and $\delta_{1}$ for $\Delta=10 \mathrm{~dB}$ are given in Table I. We then plotted the approximation error as a function of $C$ for different antenna configurations. Results in Fig. 2 shows that the accuracy of our approximation increases with $\kappa$.

Furthermore, by solving the linear equations (16), (17) and $\bar{C} \approx S_{q}+S_{p_{1}}+S_{p_{2}}$, we obtain $S_{q}, S_{p_{1}}$ and $S_{p_{2}}$ such that

$$
\begin{aligned}
S_{q} & \approx 0.5\left(\bar{C}+q \eta_{1} \ln \left(\cosh \left(\bar{C} /\left(q \eta_{1}\right)\right)\right)\right) \\
S_{p_{1}} & \approx \frac{0.5\left[\bar{C}-q \eta_{1} \ln \left(\cosh \left(\bar{C} /\left(q \eta_{1}\right)\right)\right)\right]}{1+\Psi_{p, q}} \\
S_{p_{2}} & \approx \frac{0.5\left[\bar{C}-q \eta_{1} \ln \left(\cosh \left(\bar{C} /\left(q \eta_{1}\right)\right)\right)\right] \ln \left(\Psi_{p, q}\right)}{1+\Psi_{p, q}}
\end{aligned}
$$

for $\kappa \geq 2$. In addition, $S_{q}, S_{p_{1}}$ and $S_{p_{2}}$ can be formulated as

$$
\begin{aligned}
S_{q} & \approx 0.5\left(\bar{C}+q \ln \left(1+\eta_{0}\left[\cosh \left(\bar{C} /\left(q \eta_{1}\right)\right)^{\eta_{1}}-1\right]\right)\right) \\
S_{p_{1}} & \approx \frac{0.5\left[\bar{C}-q \ln \left(1+\eta_{0}\left[\cosh \left(\bar{C} /\left(q \eta_{1}\right)\right)^{\eta_{1}}-1\right]\right)\right]}{1+\Psi_{p, q}} \\
S_{p_{1}} & \approx \frac{0.5\left[\bar{C}-q \ln \left(1+\eta_{0}\left[\cosh \left(\bar{C} /\left(q \eta_{1}\right)\right)^{\eta_{1}}-1\right]\right)\right] \ln \left(\Psi_{p, q}\right)}{1+\Psi_{p, q}}
\end{aligned}
$$

when $1 \leq \kappa<2$. Next, $\tilde{f}_{u}^{-1}(C)$ in (13) is obtained by substituting $S_{q}, S_{p_{1}}$ and $S_{p_{2}}$ (equations (18) or (19)). Our CFA for the EE-SE trade-off of the uplink of the 2BS-DMIMO system is then obtained by inserting $f_{u}^{-1}(C) \approx \tilde{f}_{u}^{-1}(C)$ in (4). In Fig. 3, we compared our uplink CFA obtained from equations (4) and (13) with the Monte-Carlo simulations for various $p \times q$ antenna configurations. As it can be observed, the results clearly show the tight fitness of our CFA with the Monte-Carlo simulations, hence, it is a graphical illustration of the accuracy of our uplink CFA. 
TABLE I

PARAMETERS $\eta_{0}, \eta_{1}, \delta_{0}$ AND $\delta_{1}$ AS A FUNCTION OF $\kappa \mid 1 / \kappa$ AT $\Delta=10 \mathrm{~dB}$

\begin{tabular}{|l||l|l|l|l|l|l|l|l|}
\hline$\kappa \mid 1 / \bar{\kappa}$ & 1 & $6 / 5$ & $3 / 2$ & $5 / 3$ & 2 & $9 / 4$ & 3 & 10 \\
\hline$\eta_{0}$ & 0.175 & 0.228 & 0.278 & 0.317 & - & - & - & - \\
\hline$\eta_{1}$ & 0.95 & 0.86 & 0.73 & 0.77 & 2.220 & 2.113 & 1.916 & 1.569 \\
\hline$\delta_{0}$ & 0.33 & 0.36 & 0.38 & 0.39 & 0.41 & 0.42 & 0.43 & 0.39 \\
\hline$\delta_{1}$ & 0.049 & 0.0298 & 0.0169 & 0.0131 & 0.0085 & 0.0065 & 0.0035 & 0.0003 \\
\hline
\end{tabular}

\section{B. EE-SE Trade-off CFA for the Downlink of $2 B S$-DMIMO}

The closed-form expression for the ergodic capacity per unit bandwidth in the downlink of DMIMO can be expressed as

$$
\begin{aligned}
C \approx \tilde{f}_{d}(\gamma)=\frac{1}{\ln (2)}[ & q \ln \left(1+\alpha_{2}^{2} P\left(\Delta u_{1}+u_{2}\right)\right)+p \ln \left(1+\bar{\kappa} \alpha_{2}^{2} \Delta P w\right) \\
& \left.+p \ln \left(1+\bar{\kappa} \alpha_{2}^{2} P w\right)-q \alpha_{2}^{2} P w\left(\Delta u_{1}+u_{2}\right)\right](20)
\end{aligned}
$$

in bits/s/Hz, where $\bar{\kappa}=\frac{q}{p}, u_{i}, i \in\{1,2\}$ and $w$ are the unique solution to the following equations:

$$
\begin{array}{rlrl}
u_{i} & =\left(1+\bar{\kappa} \alpha_{i}^{2} P w\right)^{-1} & i=1,2 \\
w & =\left(1+\alpha_{1}^{2} P u_{1}+\alpha_{2}^{2} P u_{2}\right)^{-1} &
\end{array}
$$

Similar to the uplink scenario, we define $g=\alpha_{2}^{2} P u_{2}(\Delta x+$ $1), d_{1}=\bar{\kappa} \Delta \alpha_{2}^{2} P w$ and $d_{2}=\bar{\kappa} \alpha_{2}^{2} P w$. Moreover, we define $\bar{g}=2 g+1, \bar{d}_{1}=2 d_{1}+1$ and $\bar{d}_{2}=2 d_{2}+1$. By following the same approach as in the uplink scenario, we can express (20) as in (8) and, hence, we can easily show that

$$
\bar{g}\left[\bar{d}_{2}+\left(\frac{\bar{d}_{1}}{\Delta}-\frac{1}{\Delta}+1\right)\right]=2\left[2 \alpha_{2}^{2} \gamma(\Delta x+\bar{\kappa}+1)+1\right],
$$

which results in the following approximation for $f_{d}^{-1}(C)$ in (5)

$$
\tilde{f}_{d}^{-1}(C)=\frac{-2+\left[1+\frac{1}{W_{0}\left(g_{q}\left(S_{q}\right)\right)}\right]\left[\left(1+\frac{1}{W_{0}\left(g_{p}\left(S_{p_{1}}\right)\right)}\right)+\frac{1}{\Delta}\left(2+\frac{1}{W_{0}\left(g_{p}\left(S_{p_{2}}\right)\right)}\right)-1\right]}{4 \alpha_{2}^{2}(\Delta x+\bar{\kappa}+1)} .
$$

Using a similar approach as in the uplink case, the closed-form expression for the downlink of 2BS-DMIMO can be obtained by expressing $S_{q}, S_{p_{1}}$ and $S_{p_{2}}$ as a function of $C, p$ and $q$. By utilizing the parametric expression given in (16) and (17) for $S_{q}-\left(S_{p_{1}}+S_{p_{2}}\right)$ and $\Psi_{p, q} \approx \frac{S_{p_{2}}}{S_{p_{1}}}$, respectively, we obtain the expressions of $S_{q}, S_{p_{1}}$ and $S_{p_{2}}$ given in (18) and (19), for $1 / \bar{\kappa} \geq 2$ and $1 \leq 1 / \bar{\kappa}<2$, respectively. Our CFA for the EE-SE trade-off of the downlink of the 2BS-DMIMO system is then obtained by inserting $f_{d}^{-1}(C) \approx \tilde{f}_{d}^{-1}(C)$ in (5). In Fig. 4, we demonstrate graphically the accuracy of the EE-SE CFA in the downlink of 2BS-DMIMO system for various $q \times p$ antenna configurations.

\section{ENERGY EFFICIENCY ANALYSIS OF DMIMO}

In this section, we utilize our CFA of the EE-SE tradeoff for the downlink of 2BS-DMIMO to evaluate the impact of the antenna configurations on the EE gain of the 2BSDMIMO over MIMO system when considering a realistic power consumption model (PCM) as the ones in [12] and [13]. Combining these two linear PCMs, we obtain that the realistic total power $P_{T}=p \Gamma\left(\frac{M P}{p \mu_{P A}}+P_{s p}\right)+P_{b h}$, where $M=2$, $\mu_{P A}, P_{s p}$ and $P_{b h}$ denotes the power amplifier efficiency, signal processing power and the additional backhauling induced

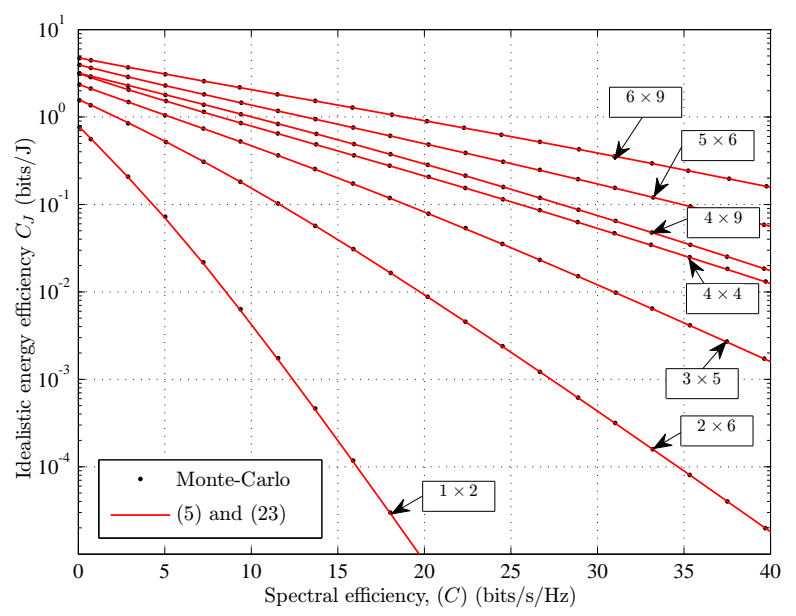

Fig. 4. EE-SE of the Downlink of 2BS-DMIMO

power for supporting DMIMO, respectively. In addition, the parameter $\Gamma=\left(1+c_{d c}\right)\left(1+c_{m s}\right)\left(1+c_{c}\right)$ accounts for the DC-DC, main supply and cooling losses i.e. $c_{d c}, c_{m s}$ and $c_{c}$ respectively. The power $P_{s p}$ can be obtained from [12] such that

$$
P_{s p}=p_{s p}\left((0.9-v)+0.1 M+M^{2} v\right) .
$$

where $p_{s p}$ is the base value of the signal processing power and $\left((0.9-v)+0.1 M+M^{2} v\right)$ represents the additional processing cost as a result of joint processing. Note that $v \%$ (where $v$ is between 1 and 10) of $p_{s p}$ is used for MIMO processing, and we assume that $v=5$. The additional backhaul power $P_{b h}$ is given by $P_{b h}=\frac{C_{d}}{C_{b h}} M p_{b}$ Watts, where $C_{b h}$ is the capacity of the backhaul link with a dissipation power $p_{b}$. Consequently, the EE-SE trade-off in the downlink of DMIMO can be reexpressed as

$$
\tilde{C}_{J_{d}}=\frac{S}{N_{0}}\left[\frac{2 \tilde{f}_{d}^{-1}(C) \Gamma}{\mu_{P A}}+\frac{p \Gamma P_{s p}+P_{b h}}{N}\right]^{-1}
$$

when considering a realistic PCM. We set the parameters in (25) by using the values related to the LTE system in Table 1 of [13], where $p_{s p}=P_{R F}+P_{B B}=42.5$ Watts. In addition, we assume that $\alpha_{1}^{2}=1, \alpha_{2}^{2}=0.1$ (such that $\Delta=10 \mathrm{~dB}$ ) and $N=1$ and the parameters in Table I unless otherwise stated.

In Fig. 5, we utilize our CFA of equation (25), for evaluating the impact of various antenna configurations on the EE of the 2BS-DMIMO system. The results clearly indicate that given a certain SE target, their exits an antenna setting that maximizes the EE. In addition, we observe that in terms of the realistic EE, having $p>q$ is not desirable, i.e., we obtain a higher EE for $\bar{\kappa}=1$ than for $\bar{\kappa}=\frac{1}{2}$. Increasing $p$ beyond $q$ does not give significant increase in the downlink capacity, however, a significant increase in power consumption is experienced.

In Fig. 6, we utilize our downlink EE-SE trade-off CFA for comparing the EE of DMIMO with that of MIMO system. We define the EE gain $G_{E E}$ as $G_{E E}=\tilde{C}_{J, D M I M O} / \tilde{C}_{J, M I M O}$, 


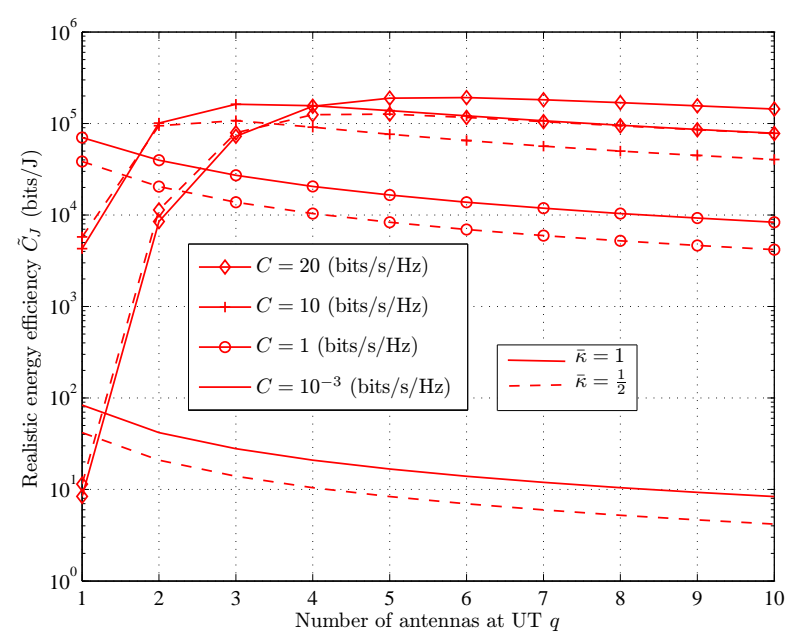

Fig. 5. Effect of number of antennas on the realistic $E E\left(\tilde{C}_{J_{d}}\right)$

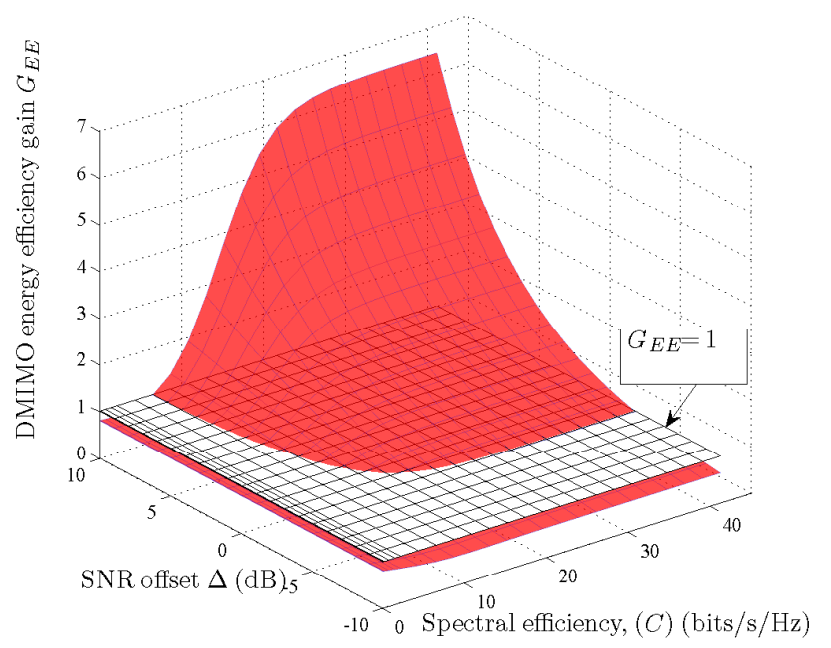

Fig. 6. Realistic EE gain of the downlink of a 2BS-DMIMO, $p=2$ and $q=2$

where $\tilde{C}_{J, D M I M O}$ is given in (25) and $\tilde{C}_{J, M I M O}$ is obtained from [3]. The PCM for MIMO can be obtained by setting $M=1, P_{b h}=0$ and $v=0$ in $P_{T}$. In order to make the comparison fair we assume an average channel gain for the MIMO system of $\alpha_{1}=1$ while we assume for the DMIMO that $\alpha_{1}=1$ and $\alpha_{2}$ varies between $\sqrt{10^{-1}}$ to $\sqrt{10}$. We obtain the values of $\eta_{0}, \eta_{1}, \delta_{0}$ and $\delta_{1}$ by numerical search. Our results indicate that at low SE, the MIMO scheme outperforms the 2BS-DMIMO over all channel offset range. However, at high SE, the DMIMO scheme performs better when the channel gain of its other link, i.e., $\alpha_{2}^{2} \succsim \alpha_{1}^{2}$, which include scenarios where both channels are not in deep fade and scenarios where a better channel than the MIMO channel can be exploited by DMIMO i.e., very good macro diversity gain.

\section{CONCLUSion}

In this paper, we have derived an accurate closed-form approximation of the EE-SE trade-off for both the uplink and downlink of the 2BS-DMIMO system over the Rayleigh fading channel and also show its accuracy over a wide range of SE and numerous antenna configurations. We then utilized our CFA to analyze the effect of using multiple antennas on the EE while considering a realistic PCM. It was revealed that their exists an antenna setting that maximizes the EE. Furthermore, our CFA was also used for assessing the EE gain of the 2BS-DMIMO over MIMO system. The DMIMO scheme can provide significant gain in terms of EE, especially when the MIMO links are in deep fade. In the future, we would extend our method for defining a more generic DMIMO system and deriving a more compact CFA of its EE-SE trade-off for both the uplink and downlink scenarios.

\section{ACKNOWLEDGMENT}

This work has been done within joint project, supported by Huawei Tech. Co., Ltd, China.

\section{REFERENCES}

[1] Y. Chen, S. Zhang, S. Xu, and G. Y. Li, "Fundamental trade-offs on green wireless networks," IEEE Commun. Mag., vol. 49, no. 6, pp. 3037, 2011.

[2] H. Kwon and T. Birdsall, "Channel capacity in Bits per Joule," IEEE J. Ocean. Eng., vol. 11, no. 1, pp. 97-99, Jan. 1986.

[3] F. Héliot, O. Onireti, and M. A. Imran, "An accurate closed-form approximation of the energy efficiency-spectral efficiency trade-off over the MIMO Rayleigh fading channel," in Proc. IEEE Int Communications Workshops (ICC) Conf, 2011, pp. 1-6.

[4] F. Héliot, M. A. Imran, and R. Tafazolli, "On the energy efficiencyspectral efficiency trade-off over the MIMO Rayleigh fading channel," to appear in IEEE Transactions on Communications.

[5] O. Onireti, F. Heliot, and M. A. Imran, "On the energy efficiency-spectral efficiency trade-off in the uplink of comp system," IEEE Trans. Wireless Commun., vol. 11, no. 2, pp. 556-561, 2012.

[6] D. Wang, X. You, J. Wang, Y. Wang, and X. Hou, "Spectral efficiency of distributed MIMO cellular systems in a composite fading channel," in Proc. IEEE Int. Conf. Communications ICC '08, 2008, pp. 1259-1264.

[7] X.-H. You, D.-M. Wang, B. Sheng, X.-Q. Gao, X.-S. Zhao, and M. Chen, "Cooperative distributed antenna systems for mobile communications," IEEE Wireless Commun. Mag., vol. 17, no. 3, pp. 35-43, 2010.

[8] D. Aktas, M. N. Bacha, J. S. Evans, and S. V. Hanly, "Scaling results on the sum capacity of cellular networks with MIMO links," IEEE Trans. Inf. Theory, vol. 52, no. 7, pp. 3264-3274, Jul. 2006.

[9] F. Héliot, R. Hoshyar, and R. Tafazolli, "An accurate closed-form approximation of the distributed MIMO outage probability," IEEE Trans. Wireless Commun., vol. 10, no. 1, pp. 5-11, 2011.

[10] F. Héliot, M. A. Imran, and R. Tafazolli, "Energy efficiency analysis of idealized coordinated multi-point communication system," in Proc. IEEE 73rd Vehicular Technology Conf. (VTC Spring), 2011, pp. 1-5.

[11] N. C. Beaulieu and F. Rajwani, "Highly accurate simple closed-form approximations to lognormal sum distributions and densities," IEEE Commun. Lett., vol. 8, no. 12, pp. 709-711, 2004.

[12] A. J. Fehske, P. Marsch, and G. P. Fettweis, "Bit per Joule efficiency of cooperating base stations in cellular networks," in Proc. IEEE Globecom, Miami, USA, Dec. 2010.

[13] G. Auer et al, "How much energy is needed to run a wireless network?" IEEE Wireless Commun. Mag., vol. 18, no. 5, pp. 40-49, 2011.

[14] W. Roh and A. Paulraj, "Outage performance of the distributed antenna systems in a composite fading channel," in Proc. IEEE 56th Vehicular Technology Conf. (VTC Fall), vol. 3, 2002, pp. 1520-1524.

[15] R. M. Corless, G. H. Gonnet, D. E. G. Hare, D. J. Jeffrey, and D. E. Knuth, "On the LambertW function," Adv. Comput. Math., vol. 5, p. 329359, 1996. 